\title{
Research on Visualization of Three-Dimensional Vectorization of Flow Field
}

\author{
Yulu Pan, Shenhua Yang*, Yongfeng Suo and Guoquan Chen \\ Navigation College, Jimei University, Jiageng Road 1st, Jimei Distract, Xiamen City, Fujian Province, China \\ "Corresponding author
}

\begin{abstract}
In order to support the comprehensive analysis of virtual simulation and scientific visualization of navigation environment in marine simulator, this paper carried out the visualization analysis of three-dimensional vector field data of flow field according to the space-time characteristics of river data The paper analyzed the characteristics of the flow field data, obtained the results of dynamic three-dimensional streamline visualization of the flow field data through the calculation and display of streamline in the flow field, and completed the preliminary research on the virtual simulation of the stream field in the voyage environment.
\end{abstract}

Keywords—visualization; interpretation; three-dimensional

\section{INTRODUCTION}

At present, the simulation of navigation environment of marine simulator is mainly the research of automatic generation technology of meteorological factors and hydrological factors, which displays the three-dimensional visual scene about wind, wave, flow, fog, rain, snow, sky and other natural phenomena. Obviously, the visualization of the flow field is to be accomplished first by the visual display of the stream. The visualization of flow field is a typical branch of scientific visualization, and its goal is to provide a suitable visual means to display the flow details and features in the data so that it can be easily identified and analyzed. The visualization of flow field is mainly computed by Navier-stokes equation. Streamline means a curve in the flow field at a particular time, in which all the velocity vectors of all the particles on the curve are tangent to the curve. In the practical application process, the researchers usually construct the streamline by the numerical integration method and the two-stream function method.

The numerical integration method is characterized by simple calculation and discrete thinking in fluid calculation, and it needs to solve two major problems, such as the selection of numerical integration order and the choice of integral step. The two-stream function method converts the fluid defined in a cell into a fluid represented by two three-dimensional flow functions, and the intersection line of two flow functions is streamline, and a constant solution of each set of flow functions corresponds to a streamline. The greatest advantage of this method is that it satisfies the conservation of fluid quality and avoids the error, but the disadvantage is that the computational capacity is large. These two methods are used in two different ways to express streamline, and they have their own characteristics and advantages.
At present, the difficulties of visualization of the flow field are mainly as follows:

\section{A. Large Data Set}

The Flow field data is usually vector data with direction, and it has space-time characteristics, which makes the volume of flow field data much larger than scalar data. In addition, the high precision data collection and the necessity of large time scale analysis make the research of stream data move toward large data set. And scientists prefer to see the results in real time interactively.

\section{B. Streamline Placement}

Because the mainstream method of flow visualization is based on the geometric seed point integral, the position of the seed point is directly related to the final result of the flow field. The researchers would prefer to show the areas of interest or feature structures interactively.

\section{Calculating Time and Irregular Grids}

Another challenge is computational efficiency and irregular grids. Most of the visualization calculations are based on the geometric particle tracking method [1], which is used to integrate the flow field velocity as a streamline tangent. This calculation is particularly difficult in three-dimensional unstructured grids, which can take a lot of computational time. The trend in recent years is to move computing from the CPU to the GPU to compute [2].Although the result is similar, it greatly improves the rendering efficiency, and also gives us a new way of thinking.

\section{Perception}

The elevation of awareness is eagerly concerned with the visualization of $3 \mathrm{D}$ and $4 \mathrm{D}$ vector fields and the visualization of multivariate datasets. Especially in the three-dimensional vector field streamline visualization, excessive streamline can lead to occlusion and visual clutter, too little streamline will lose important details. Therefore, an optimal balance mechanism is very important to weigh the perception and the amount of information. It is also one of the difficulties in the three-dimensional spatial streamline interaction.

\section{ChaRACTERISTICS OF FLOW FIELD DATA}

At present, the most common flow field data are pattern data, which is to solve the practical problems such as ocean wave numerical simulation, tidal exchange numerical simulation and current numerical simulation by numerically solving hydrodynamic equations. Computational techniques 
such as parallel operations and cluster operations provide the possibility for large-scale computation of complex problems. At present, the common ocean numerical models are POM (Princeton Ocean Model), FVCOM(An Unstructured Grid, Finite-Volume Coastal Ocean Model), HAMSOM(Hamburg Shelf Ocean Model), HYCOM(Hybrid Coordinate Oceanic Circulation Model),etc. [3] The data products of POM and FVCOM model adopt sigma coordinate in the vertical direction to fit the complicated seabed topography. In the vertical direction, the HAMSOM mode adopts vertical stratified pattern of depth z-coordinate, and the HYCOM adopts vertical fitting coordinates (such as density coordinate, sigma coordinate and $\mathrm{z}$ coordinate mixing). This shows that different numerical model output data product structure is not the same. And the irregular ocean data in the flow field data is very common, which is necessary for visualization research.

Vertical z-coordinate: The data structure is clear. The distribution of the information is generally evenly distributed in the three direction axis $\mathrm{x}$ axis (horizontal), $\mathrm{Y}$ axis (longitudinal axis), and $\mathrm{Z}$ axis (vertical axis). Any two of the three axes can determine a plane, called a coordinate plane, such as a XOY plane, a YOZ plane, a XOZ plane. This kind of data organization is easy to use the graphical method to sample and compute, the control grid is also more linear grid, which called regular grid data.

Vertical $\sigma$ (sigma) coordinates: the sigma coordinate system makes the uneven surface a contour of the new coordinate system, which is also called the terrain coordinate system. Sigma coordinates are widely used in the simulation of fluids, especially in river mouths and shoals. On the XY coordinate plane, each grid is a regular quadrilateral and an orthogonal grid line with curves. The corresponding nodes on different layers usually have the same XY coordinates. And the gridlines are perpendicular and parallel to the $\mathrm{z}$ axis in the vertical direction. The Fig. I(a) is a distribution rule for sigma coordinates, and the Fig. I(b) is a schematic diagram of an irregular grid [4].

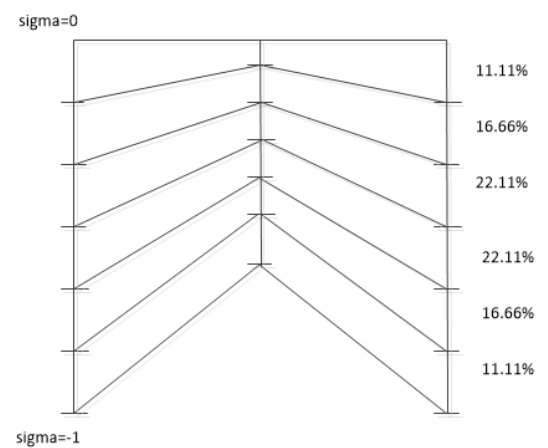

(a)

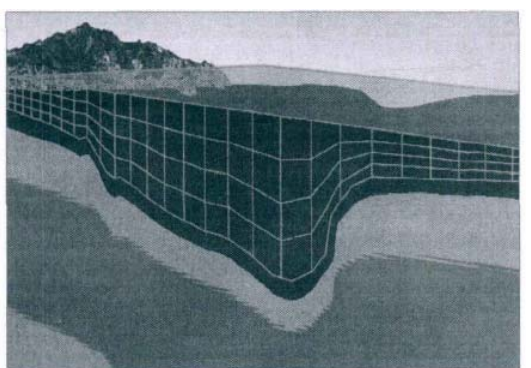

(b)

FIGURE I. (A). DIAGRAM OF VERTICAL $\Sigma$ (SIGMA) COORDINATES (B). DIAGRAM OF VERTICAL $\Sigma$ (SIGMA) COORDINATES

\section{INTERPRETATION OF FLOW FIELD DATA}

The data of flow field visualization simulation mainly comes from the data of the actual observation point. At present, there is not a large scale synchronous observation method of space-time. And these observational data are often discrete in the process of collection. The visualization of the threedimensional field of the general marine hydrological environment is continuous; therefore, in order to visualize the marine hydrological data, we must find a way to obtain the data values of each point in the water body of the study area.

Therefore, we need to make full use of these finite and scattered data, and use a reasonable interpolation algorithm to calculate the continuous distribution of ocean data as accurately as possible. In this paper, the distance inverse weighted method is used.

The distance inverse weighted method is a local interpolation method, which is based on the spatial similarity principle of data distribution: The nearer point in the space position, the more likely to have similar characteristics, the more close the attribute value, so that the attribute value of a position point can be obtained by weighting the value of the known point around it. In the process of calculation, give a larger weight to the sample point closer to the interpolation point. The calculation formula is as follows:

$$
v\left(x_{o}, y_{0}, z_{0}\right)=\frac{\sum v_{i} d_{i}^{-r}}{\sum d_{i}^{-r}} \quad i=1,2,3, \cdots \cdots, n
$$

In the formula, $v$ represents the attribute value of the insertion point, vi is the attribute value of the ith point which is searched by the insertion point, and di is the distance from the ith sample point to the insertion point for the insertion point. $r$ is often taken as 1 , which is, the reciprocal of the distance as the weight, or $r=2$ (3), which uses the reciprocal of the distance square (cubic) as the weight coefficient.

The inverse weighted method of three-dimensional distance is extended on the basis of two dimensions. It expands the planar window area of the two-dimensional local interpolation into a form, which can be a cube or a circular sphere. The specific method is: Select the regular point which distributed throughout a three-dimensional space area as the insertion point, and the value of the insertion point is determined by the data of the known sample point within a form region around the 
insertion point. In this process, the adjacent space body of the insertion point is like an active window moving along with the processing of each point to be inserted.

\section{DynAmic ThreE-Dimensional Streamline VISUALIZATION OF FLOW FIELD DATA}

According to the spatial structure of the flow field data, we establish the hexahedral control grid, such as Fig. II (a), the resolution of the hexahedral grid is 472 408. Each hexahedral unit can be retrieved from the ordering of the data, as shown in Fig. II (b) is the hexahedral $(\mathrm{i}, \mathrm{j}, \mathrm{k})$ in the data space, so it is easy to locate the corresponding attribute value in the data space. The direction of longitude increment along the $\mathrm{AB}$ direction, the direction of latitude increasing along the $\mathrm{BC}$ direction, and the direction of depth increment along AE.

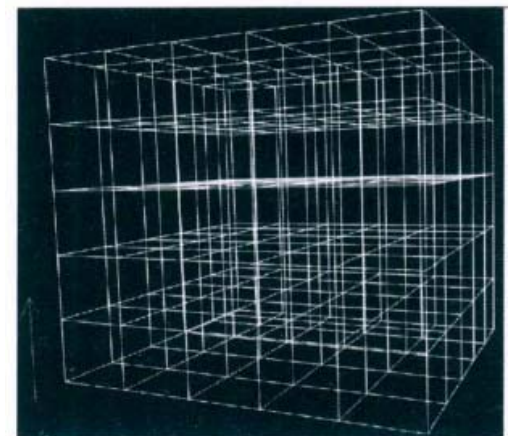

(a)

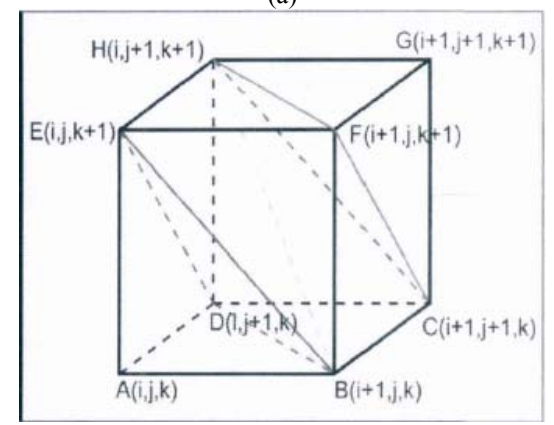

(b)

FIGURE II. (A) HEXAHEDRAL GRID DIAGRAM (B) HEXAHEDRAL UNIT SPLIT INTO SIX TETRAHEDRON

According to the segmentation method of figure II [5], the hexahedral ABCDEFGH is divided into AEDB, DB, EBFH, FCGH, HBCD, HFCB six tetrahedron, and then numbered from 0-5 under Hexahedral ABCDEFGH. From the graph, it is easy to infer the coplanar tetrahedron or the tetrahedron index number on the basis of the subscript index number, so as to facilitate the location of the point and the interpolation of the velocity.

Then the paper fills the whole region with a uniform series of lines according to the given situation, and it is generally considered that the placement strategy of the long streamline is better than that of the short streamline. According to the theory and characteristics of streamline and flow field, we know that the point direction is tangent to the vector of the flow field at that point. It is concluded that streamline placement can be determined by determining the relative position of the seed point in the flow field.

The concrete idea: First, we establish hexahedral grid based on the data resolution and streamline density requirements, initialize all grid cells to empty, make invalid values and boundary areas be full, and then find the largest connected area in the contiguous and vertical flow field, the central point of which is the position of the seed point. According to the flow field data forward integral, we take the data back to the inverse of the integration with the midpoint as the starting point, set the grid to full when the streamline go through, and continue to find the largest connected region to put seed points in empty remaining state of region, until the maximum connected value is less than a certain threshold. Implementation methods such as equation 2 and 3, this method can maximize the production of long streamline, calculate quickly, operate easily, make streamline distribution more uniform, and maintain the topological structure better.

$$
\begin{aligned}
& \operatorname{Seed}(i, j, k)=\operatorname{Pas}(\operatorname{Max}(D(i)+D(j)+D(k))) \\
& D(x)=\sum_{k++}^{n} c \_N u m\left(e \_c u b e\right)+\sum_{k-} c \_N u m\left(e \_a b e\right)
\end{aligned}
$$

This paper determines the relation between the point and tetrahedron ny the volume coordinate method, which is similar to the method of vector algorithm. Firstly, we position the hexahedron, then position the tetrahedron according to the vector relation of volume coordinate and tetrahedron. Given P $(\mathrm{x}, \mathrm{y}, \mathrm{z})$, according to the finite element theory, the volume coordinate of $\mathrm{P}$ in tetrahedron $\mathrm{ABCD}$ is as equation (4). The molecule is the new tetrahedron volume formed by the point and one of the planes, the tetrahedron volume is calculated as $\mathrm{V}_{\mathrm{ABCD}}=$ fabs $\left(\mathrm{AB}^{*}(\mathrm{AC} * \mathrm{AD})\right) / 6$, and the results may be negative. [4]

$$
C_{A}=\frac{V_{B C D P}}{V_{A B C D}} C_{B}=\frac{V_{A C D P}}{V_{A B C D}} C_{C}=\frac{V_{A D B P}}{V_{A B C D}} C_{D}=\frac{V_{A B C P}}{V_{A B C D}}
$$

It is necessary to obtain the speed of the line when we need to solve the streamline, and we usually obtain the speed of each point on the curve by the discrete tetrahedron using the difference value method.

This paper integer streamline by the method of dynamic step length [6], and consider the change of vector deflection angle and the inradii radius of tetrahedron when choosing the step size.

\section{A. Determine the Integral Step Size According to the Change of Flow Direction}

If the streamline on the light before and after the speed vector between the deflection angle is too large,it indicates that the streamline deflection is more intense. We believe that the information of the which is richer, so we need a higher fineness of streamline effect, and need to reduce the integral step. 


\section{B. Determine the Integral Step Size According to the Escribed Radius of the Body}

If the integral step size is too little, the integral process may be complex in the same tetrahedron, which will greatly affect streamline generation efficiency. At this time, we take the in-radii radius of tetrahedron as the reference standard, and determine the integral step, so as to carry on the integral circulation.

\section{SUMMARY}

Through the analysis and interpretation of flow field data and the three-dimensional streamline generation technology based on tetrahedral mesh, this paper makes a preliminary analysis of the flow field's three-dimensional vectorization, and carries out the first step of simulation study of the convection field, and hopes to realize the real-time rendering of three-dimensional flow field.

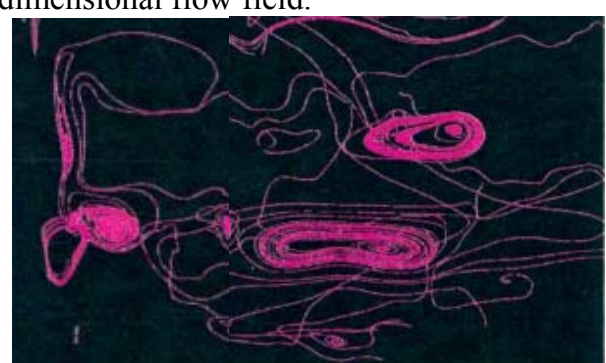

FIGURE III. STREAMLINE EFFECT DIAGRAM

\section{ACKNOWLEDGMENT}

In writing this paper, I have benefited from the presence of my teachers. They generously helped me collect data what I need and made many invaluable suggestions. Particularly, I am deeply indebted to Professor Shenhua Yang, my supervisor who guided me throughout my writing of this thesis. This study was supported by the National Natural Science Foundation of China(51579114).

\section{REFERENCES}

[1] Weiskopf D; Schramm F; Erlebacher G. et al. Particle and texture based spatiotemporal visualization of time-dependent vector fields. VIS 05. IEEE Visualization, 2005.639 - 646

[2] Burger K. Schneider J, Kondratieva P, et al. Interactive Visual Exploration of Unsteady 3D Flows. EuroVis.2007.251-258

[3] Shah M A, Konttinen J, Pattanaik S.Caustics Mapping: An image-space technique for real-time caustics. Visualization and Computer Graphics .IEEE Transactions on Visualization and Computer Graphics $13,2,272-280$

[4] Pengbo JI. Research on ocean Simulation and visualization of threedimensional flow field based on i4ocean2.0. Ocean University of China, 2015.

[5] Zhang W, Zhang M, Sun B. Multiresolution Streamline Placement for 2D Flow Fields. Computational Intelligence and Security(CIS). Seventh International Conference on.IEEE.2011.1174-1178

[6] GuangYang, Shiming Cheng. A three dimensional streamline structure based on tetrahedron. Journal of Beihang University.2008.34(9):10611064 